\title{
INERTIAL MANIFOLDS AND STABILIZATION OF NONLINEAR BEAM EQUATIONS WITH BALAKRISHNAN-TAYLOR DAMPING
}

\author{
YUNCHENG YOU
}

\begin{abstract}
In this paper we study a hinged, extensible, and elastic nonlinear beam equation with structural damping and Balakrishnan-Taylor damping with the full exponent $2(n+\beta)+1$. This strongly nonlinear equation, initially proposed by Balakrishnan and Taylor in 1989, is a very general and useful model for large aerospace structures. In this work, the existence of global solutions and the existence of absorbing sets in the energy space are proved. For this equation, the feature is that the exponential rate of the absorbing property is not a global constant, but which is uniform for the family of trajectories starting from any given bounded set in the state space. Then it is proved that there exists an inertial manifold whose exponentially attracting rate is accordingly non-uniform. Finally, the spillover problem with respect to the stabilization of this equation is solved by constructing a linear state feedback control involving only finitely many modes. The obtained results are robust in regard to the uncertainty of the structural parameters.
\end{abstract}

\section{Introduction AND Formulation}

The objective of this paper is to study the following initial-boundary value problem for a nonlinear beam equation,

$$
\begin{aligned}
u_{t t}+ & \alpha u_{x x x x}-\delta u_{x x t} \\
& -\left\{a+b \int_{0}^{1}\left|u_{x}(t, \xi)\right|^{2} d \xi+q\left[\int_{0}^{1}\left(u_{x} u_{x t}\right)(t, \xi) d \xi\right]^{2(n+\beta)+1}\right\} u_{x x} \\
= & f, \quad \text { for }(t, x) \in \mathcal{R}^{+} \times(0,1), \\
& u(t, 0)=u_{x x}(t, 0)=u(t, 1)=u_{x x}(t, 1)=0, \quad \text { for } t \geq 0, \\
& u(0, x)=u_{0}(x), u_{t}(0, x)=u_{1}(x), \quad \text { for } x \in[0,1] .
\end{aligned}
$$

1991 Mathematics Subject Classification. 35B40, 35L75, 73K05, 93D15.

Key words and phrases. Inertial manifold, stabilization, nonlinear beam equation, dissipative solution semigroup, Balakrishnan-Taylor damping.

Received: February 4, 1996 
Here $u(t, x)$ is the transverse deflection of the beam. All the parameters $\alpha, \delta, b$, and $q$ are assumed to be positive constants, but $a \in \mathcal{R}$. The term $-\delta u_{x x t}$ represents the structural damping, $\left[a+b\left\|u_{x}\right\|^{2}\right] u_{x x}$ is the tension due to the extensibility, and the last term on the left-hand side is called the Balakrishnan-Taylor damping. The parameter $\beta$ satisfies $0 \leq \beta<1 / 2$ and $n \geq 0$ is an integer. The function $f(t, x)$ stands for an external input which practically may be a control function. The boundary conditions correspond to hinged endpoints. This model equation of nonlinear beams was initially proposed by Balakrishnan and Taylor in 1989 (cf. [1] and [2]). The original motivation for studying this model seemed to solve the spillover problem, namely, to design a feedback control function $f$ that involves only finitely many modes in order to achieve a high performance of the closed-loop system, such as a robust and exponential stabilization of the system when there might be some uncertainty in the values of the parameters.

In this paper we first analyze the global dynamics of the uncontrolled equation and prove the existence of inertial manifolds. Then, based on this analysis and result, we provide a solution to the spillover problem by constructing an implementable feedback control which involves only finitely many modes and is robust with respect to parameter uncertainty.

An initial result on the existence of a flat inertial manifold for the dynamics of a rotating beam was obtained in [3]. The long time behavior and global dynamics of a simpler version of Equation (1) with $q=0$ and damping $\delta u_{t}$ has been studied by many authors (cf. [6], [7] and [8]). This model with a simplified exponent assumption that $n=\beta=0$ has been investigated and the affirmative results on the existence of inertial manifolds and on the finite-dimensional stabilization have been proved in [11] and [12]. More background materials in regard to infinite dimensional dynamical systems, especially the theory of global attractors, inertial manifolds, and approximate inertial manifolds, can be found in [5], [7], [9] and [10].

First of all, let us formulate this initial-boundary value problem for the uncontrolled equation

$$
\begin{aligned}
u_{t t}+ & \alpha u_{x x x x}-\delta u_{x x t} \\
& -\left\{a+b \int_{0}^{1}\left|u_{x}(t, \xi)\right|^{2} d \xi+q\left[\int_{0}^{1}\left(u_{x} u_{x t}\right)(t, \xi) d \xi\right]^{2(n+\beta)+1}\right\} u_{x x} \\
& =0
\end{aligned}
$$

into an abstract semilinear evolution equation and consider the existence, uniqueness, and regularity of local solutions.

Let $H=L^{2}(0,1)$ with norm and inner-product denoted by $|\cdot|$ and $\langle$,$\rangle ,$ respectively. Define a linear operator $A: D(A) \rightarrow H$ by

$$
A \phi=\frac{d^{4} \phi}{d x^{4}}, \quad \text { for } \phi \in D(A), \text { with }
$$




$$
D(A)=\left\{\phi \in H^{4}(0,1): \phi(0)=\phi^{\prime \prime}(0)=\phi(1)=\phi^{\prime \prime}(1)=0\right\},
$$

where, in general, the derivatives are taken in the distributional sense. Here for $\phi \in D(A)$ these derivatives are consistent with the usual derivatives. This closed linear operator $A$ is densely defined, self-adjoint, and positive definite. It has compact resolvent $A^{-1}$. The spectrum $\sigma(A)$ consists of the simple eigenvalues $\left\{\lambda_{k}=k^{4} \pi^{4}: k \geq 1\right\}$, with eigenvectors

$$
\left\{e_{k}=\sqrt{2} \sin (k \pi x): k \geq 1\right\} .
$$

By the approach of Fourier expansions, it can be shown that

$$
A^{1 / 2} \phi=-d^{2} \phi / d x^{2} \quad \text { end } \quad\left|\phi_{x}\right|^{2}=\left|A^{1 / 4} \phi\right|^{2} .
$$

Thus, Equation (2) can be formulated into a second-order semilinear evolution equation:

$$
\begin{aligned}
& \frac{d^{2} u}{d u^{2}}+ \alpha A u+\delta A^{1 / 2} \frac{d u}{d t} \\
&+\left\{a+b\left|A^{1 / 4} u\right|^{2}+q\left\langle A^{1 / 2} u, u_{t}\right\rangle^{2(n+\beta)+1}\right\} A^{1 / 2} u=0, \\
& u(0)=u_{0}, \quad u_{t}(0)=u_{1} .
\end{aligned}
$$

Let $V=D\left(A^{1 / 2}\right)$ with the norm $\|v\|=\left|A^{1 / 2} v\right| . V$ is also a Hilbert space. Define the product Hilbert space $E=V \times H$, which can be called the energy space. Similarly, define $E_{1}=D(A) \times V=D(A) \times D\left(A^{1 / 2}\right)$ with the graph norm. Then define a linear operator $G$ by

$$
G=\left(\begin{array}{cc}
0 & I_{V} \\
-\alpha A & -\delta A^{1 / 2}
\end{array}\right): D(G) \rightarrow E, \text { with } D(G)=D(A) \times V,
$$

where $I_{V}$ is the identity operator on $V$, and a nonlinear mapping $R$ by

$$
R\left(\begin{array}{c}
\phi \\
\psi
\end{array}\right)=\left(\begin{array}{c}
0 \\
-\left[a+b\left|A^{1 / 4} \phi\right|^{2}+q\left\langle A^{1 / 2} \phi, \psi\right\rangle^{2(n+\beta)+1}\right] A^{1 / 2} \phi
\end{array}\right) .
$$

Then Equation (4) can be further formulated into a first-order semilinear evolution equation:

$$
\begin{gathered}
\frac{d}{d t}\left(\begin{array}{l}
u \\
v
\end{array}\right)=G\left(\begin{array}{l}
u(t) \\
v(t)
\end{array}\right)+R\left(\begin{array}{l}
u(t) \\
v(t)
\end{array}\right), \quad t \geq 0, \\
\left(\begin{array}{l}
u(0) \\
v(0)
\end{array}\right)=\left(\begin{array}{l}
u_{0} \\
v_{0}
\end{array}\right) \in E,
\end{gathered}
$$

where $v_{0}=u_{1}$. Let $w(t)=\left(\begin{array}{c}u(t) \\ v(t)\end{array}\right)$ and $w_{0}=\left(\begin{array}{c}u_{0} \\ v_{0}\end{array}\right)$. Then Equation $(7)$ can be rewritten as

$$
\frac{d w}{d t}=G w+R(w), \quad t \geq 0, \quad w(0)=w_{0} \in E .
$$

It can be shown that the operator $-G$ is a sectorial operator and that $G$ generates an analytic contraction semigroup which will be denoted by $\{T(t): t \geq 0\}$. Moreover, $G$ has compact resolvent. The nonlinear mapping $R: E \rightarrow E$ is locally Lipschitz continuous and maps bounded sets into bounded sets. Therefore, by the standard semigroup theory, we have the following local existence and regularity result, whose proof is omitted. 
Lemma 1. For any $w_{0} \in E$, there is a $\tau=\tau\left(w_{0}\right)>0$ such that the mild solution $w(t)$ of Equation (8) with the initial condition $w(0)=w_{0}$ exists uniquely for $t \in[0, \tau]$ and

$$
w \in C([0, \tau] ; E) \cap C^{1}((0, \tau) ; E) \cap C\left((0, \tau) ; E_{1}\right) .
$$

If $w_{0} \in E_{1}$, then this mild solution is a classical solution of Equation (8) for $t \in[0, \tau]$.

\section{Global Existence of Solutions And Dissipativity}

In this section we shall simultaneously prove the global existence of mild solutions of Equation (8) and the dissipativity of the dynamical system associated with the solutions semigroup. Let us introduce the relevant concepts.

A fixed bounded set $\mathbf{N}$ is called an absorbing set for the solution semigroup $S(t), t \geq 0$, generated by the mild solutions of Equation (8), if for any given bounded set $Z$ of $E$, there exist constants $\varepsilon(Z)>0$ and $K(Z) \geq 0$ such that, for any initial data $w_{0} \in Z$,

$$
\operatorname{dist}_{E}\left(w\left(t ; w_{0}\right), \mathbf{N}\right) \leq K(Z) \exp (-\varepsilon(Z) t), \quad t \geq 0 .
$$

Theorem 2. For any $w_{0} \in E$, there exists a unique global mild solution $w(t)$ of Equation (8), $t \in[0, \infty)$, which has the regularity properties stated in Lemma 1. The associated solution semigroup $S(t), t \geq 0$, is dissipative in the sense that there exists an absorbing set in $E$.

Proof. By taking the inner product in $H$ of Equation (2) with $2 u_{t}$, we obtain

$$
\begin{aligned}
& \frac{d}{d t}\left(\left|u_{t}\right|^{2}+\alpha\left|u_{x x}\right|^{2}\right)+2 \delta\left|u_{x t}\right|^{2} \\
& \quad+\left\{a+b\left|u_{x}\right|^{2}+q\left(\frac{1}{2} \frac{d}{d t}\left|u_{x}\right|^{2}\right)^{2(n+\beta)+1}\right\} \frac{d}{d t}\left|u_{x}\right|^{2} \\
& =\frac{d}{d t}\left[\left|u_{t}\right|^{2}+\alpha\left|u_{x x}\right|^{2}+\frac{1}{2 b}\left(a+b\left|u_{x}\right|^{2}\right)^{2}\right] \\
& \left.\quad+2 \delta\left|u_{x t}\right|^{2}+q\left(\frac{1}{2} \frac{d}{d t}\left|u_{x}\right|^{2}\right)^{2(n+\beta+1)}\right)^{2(n+1)}+ \\
& =0
\end{aligned}
$$

By integrating (10) over $[0, t]$, for any $t \geq 0$, we have

$$
\begin{aligned}
\left|u_{t}\right|^{2}+ & \alpha\left|u_{x x}\right|^{2}+\frac{1}{2 b}\left(a+b\left|u_{x}\right|^{2}\right)^{2} \\
& +\int_{0}^{t}\left[2 \delta\left|u_{x t}\right|^{2}(s)+q\left(\frac{1}{2} \frac{d}{d t}\left|u_{x}\right|^{2}(s)\right)^{2(n+\beta+1)}\right] d s \\
\leq & \left|u_{1}\right|^{2}+\alpha\left|A^{1 / 2} u_{0}\right|^{2}+\frac{1}{2 b}\left(a+b\left|A^{1 / 4} u_{0}\right|^{2}\right)^{2} \\
\leq & C_{0}+C_{1}\left\|\left(u_{0}, u_{1}\right)\right\|_{E}^{4},
\end{aligned}
$$


where $C_{0}$ and $C_{1}>0$ are constants independent of the initial data $\left(u_{0}, u_{1}\right)$. Note that each term on the left-hand side of the first inequality of (11) is uniformly bounded in $t$, if the initial data $\left(u_{0}, u_{1}\right)$ belong to a given bounded subset $Z$ of $E$.

Then take the inner product in $H$ of Equation (2) with $\varepsilon u$, where $\varepsilon>0$ is an undetermined constant, to obtain

$$
\begin{aligned}
\frac{d}{d t}( & \left.\varepsilon\left\langle u_{t}, u\right\rangle+\frac{\varepsilon \delta}{2}\left|u_{x}\right|^{2}\right)+\varepsilon \alpha\left|u_{x x}\right|^{2}-\varepsilon\left|u_{t}\right|^{2} \\
& +\varepsilon\left\{a+b\left|u_{x}\right|^{2}+q\left(\frac{1}{2} \frac{d}{d t}\left|u_{x}\right|^{2}\right)^{2(n+\beta)+1}\right\}\left|u_{x}\right|^{2} \\
= & \frac{d}{d t}\left(\varepsilon\left\langle u_{t}, u\right\rangle+\frac{\varepsilon \delta}{2}\left|u_{x}\right|^{2}\right)+\varepsilon \alpha\left|u_{x x}\right|^{2}-\varepsilon\left|u_{t}\right|^{2} \\
& +\varepsilon\left\{\frac{1}{2} a b^{-1 / 2}+b^{1 / 2}\left|u_{x}\right|^{2}\right\}^{2}-\frac{1}{4} \varepsilon a^{2} b^{-1} \\
& +\varepsilon q\left(\frac{1}{2} \frac{d}{d t}\left|u_{x}\right|^{2}\right)^{2(n+\beta)+1}\left|u_{x}\right|^{2} \\
= & 0 .
\end{aligned}
$$

Adding (10) and (12), we obtain

$$
\begin{aligned}
& \frac{d}{d t}\left\{\left|u_{t}\right|^{2}+\alpha\left|u_{x x}\right|^{2}+\frac{1}{2 b}\left(a+b\left|u_{x}\right|^{2}\right)^{2}+\varepsilon\left\langle u_{t}, u\right\rangle+\frac{\varepsilon \delta}{2}\left|u_{x}\right|^{2}\right\} \\
& \quad+\left\{2 \delta\left|u_{x t}\right|^{2}+q\left(\frac{1}{2} \frac{d}{d t}\left|u_{x}\right|^{2}\right)^{2(n+\beta+1)}+\varepsilon \alpha\left|u_{x x}\right|^{2}-\varepsilon\left|u_{t}\right|^{2}\right. \\
& \left.\quad+\varepsilon\left[\frac{1}{2} a b^{-1 / 2}+b^{1 / 2}\left|u_{x}\right|^{2}\right]^{2}\right\}+\varepsilon q\left(\frac{1}{2} \frac{d}{d t}\left|u_{x}\right|^{2}\right)^{2(n+\beta)+1}\left|u_{x}\right|^{2} \\
& =\frac{1}{4} \varepsilon a^{2} b^{-1} .
\end{aligned}
$$

Note that

$$
q\left(\frac{1}{2} \frac{d}{d t}\left|u_{x}\right|^{2}\right)^{2(n+\beta+1)} \geq 0
$$

However,

$$
\varepsilon q\left(\frac{1}{2} \frac{d}{d t}\left|u_{x}\right|^{2}\right)^{2(n+\beta)+1}\left|u_{x}\right|^{2}
$$


may be negative. Define the functionals $N(t)$ and $L(t)$ by

$$
\begin{aligned}
N(t)= & 2 \delta\left|u_{x t}\right|^{2}+\varepsilon \alpha\left|u_{x x}\right|^{2}-\varepsilon\left|u_{t}\right|^{2}+\varepsilon\left[\frac{1}{2} a b^{-1 / 2}+b^{1 / 2}\left|u_{x}\right|^{2}\right]^{2} \\
& +q\left(\frac{1}{2} \frac{d}{d t}\left|u_{x}\right|^{2}\right)^{2(n+\beta)}\left[\frac{1}{2} \frac{d}{d t}\left|u_{x}\right|^{2}+\frac{\varepsilon}{2}\left|u_{x}\right|^{2}\right]^{2} \\
= & \varepsilon\left\{2 \delta \varepsilon^{-1}\left|u_{x t}\right|^{2}+\alpha\left|u_{x x}\right|^{2}-\left|u_{t}\right|^{2}+\left[\frac{1}{2} a b^{-1 / 2}+b^{1 / 2}\left|u_{x}\right|^{2}\right]^{2}\right\} \\
& +q\left(\frac{1}{2} \frac{d}{d t}\left|u_{x}\right|^{2}\right)^{2(n+\beta)}\left[\frac{1}{2} \frac{d}{d t}\left|u_{x}\right|^{2}+\frac{\varepsilon}{2}\left|u_{x}\right|^{2}\right]^{2}
\end{aligned}
$$

and

$$
L(t)=\left|u_{t}\right|^{2}+\alpha\left|u_{x x}\right|^{2}+\frac{1}{2 b}\left(a+b\left|u_{x}\right|^{2}\right)^{2}+\varepsilon\left\langle u_{t}, u\right\rangle+\frac{\varepsilon \delta}{2}\left|u_{x}\right|^{2} .
$$

By Poincaré's inequality and (3), we have $\left|u_{x t}\right| \geq\left|u_{t}\right|$ and $\left|u_{x x}\right| \geq\left|u_{x}\right| \geq|u|$. Then,

$$
\begin{aligned}
2 \varepsilon^{-1} N(t)-L(t) \geq & \left(\frac{4 \delta}{\varepsilon}-3-\varepsilon\right)\left|u_{x t}\right|^{2}+\left(\alpha-\varepsilon-\frac{\varepsilon \delta}{2}\right)\left|u_{x x}\right|^{2} \\
& +\frac{1}{2 b}\left[a+2 b\left|u_{x}\right|^{2}\right]^{2}-\frac{1}{2 b}\left(a+b\left|u_{x}\right|^{2}\right)^{2} \\
\geq & \left(\frac{4 \delta}{\varepsilon}-3-\varepsilon\right)\left|u_{x t}\right|^{2}+\left(\alpha-\varepsilon-\frac{\varepsilon \delta}{2}\right)\left|u_{x x}\right|^{2} \\
& +\left|u_{x}\right|^{2}\left(a+b\left|u_{x}\right|^{2}\right) .
\end{aligned}
$$

Now we can choose $\varepsilon>0$ sufficiently small so that

$$
\frac{4 \delta}{\varepsilon}-3-\varepsilon \geq 0, \quad \alpha-\varepsilon-\frac{\varepsilon \delta}{2} \geq 0, \quad \text { and } \frac{1}{2} \min \{1, \alpha\} \geq \varepsilon .
$$

By completing the square for the last term on the right-hand side of Inequality (16), we get

$$
2 \varepsilon^{-1} N(t)-L(t) \geq-\frac{a^{2}}{4 b} .
$$

Substitution of (17) into (13) gives rise to a differential inequality:

$$
\begin{aligned}
\frac{d}{d t} & L(t)+\frac{\varepsilon}{2} L(t) \leq \frac{\varepsilon a^{2}}{2 b}+\frac{\varepsilon^{2}}{4} q\left(\frac{1}{2} \frac{d}{d t}\left|u_{x}\right|^{2}\right)^{2(n+\beta)}\left|u_{x}\right|^{4} \\
& \leq \frac{\varepsilon a^{2}}{2 b}+C(n+\beta) \varepsilon^{2} q\left\{\left[\frac{1}{2} \frac{d}{d t}\left|u_{x}\right|^{2}\right]^{2(n+\beta+1)}+\left|u_{x}\right|^{4(n+\beta+1)}\right\} \\
& \leq \frac{\varepsilon a^{2}}{2 b}+C(n+\beta) \varepsilon^{2} q\left\{\left[\frac{1}{2} \frac{d}{d t}\left|u_{x}\right|^{2}\right]^{2(n+\beta+1)}+C_{2}\left(u_{0}, u_{1}\right)\right\}
\end{aligned}
$$


for $t \in I_{\max }$ (the maximal interval of existence), where $C(n+\beta)$ is a constant depending on $n+\beta$ and obtained by applying Young's inequality to split the product on the right-hand side of the first inequality of (18). Besides, the inequality

$$
\frac{1}{2 b}\left(a+b\left|u_{x}\right|^{2}\right)^{2} \leq C_{0}+C_{1}\left\|\left(u_{0}, u_{1}\right)\right\|_{E}^{4}
$$

is utilized to bound $\left|u_{x}\right|^{4(n+\beta+1)}$, with the constant $C_{2}\left(u_{0}, u_{1}\right)$ given by

$$
C_{2}\left(u_{0}, u_{1}\right)=\left\{\frac{\left[2 b\left(C_{0}+C_{1}\left\|\left(u_{0}, u_{1}\right)\right\|_{E}^{4}\right)\right]^{1 / 2}+|a|}{b}\right\}^{2(n+\beta+1)}
$$

Hence it follows that

$$
\begin{aligned}
L(t) \leq & L(0) \exp \left(-\frac{\varepsilon}{2} t\right)+\frac{a^{2}}{b} \\
& +C(n+\beta) \varepsilon^{2} q \int_{0}^{t}\left[\frac{1}{2} \frac{d}{d t}\left|u_{x}\right|^{2}(s)\right]^{2(n+\beta+1)} d s \\
& +C(n+\beta) \varepsilon^{2} q \int_{0}^{t} C_{2}\left(u_{0}, u_{1}\right) \exp \left(-\frac{\varepsilon}{2}(t-s)\right) d s \\
\leq & L(0) \exp \left(-\frac{\varepsilon}{2} t\right)+\frac{a^{2}}{b} \\
& +C(n+\beta) \varepsilon^{2}\left(C_{0}+C_{1}\left\|\left(u_{0}, u_{1}\right)\right\|_{E}^{4}\right) \\
& +2 C(n+\beta) \varepsilon q C_{2}\left(u_{0}, u_{1}\right),
\end{aligned}
$$

where the bound for the first integral term comes from the property (11). Note that

$$
\begin{aligned}
L(t) & =\left|u_{t}\right|^{2}+\alpha\left|u_{x x}\right|^{2}+\frac{1}{2 b}\left(a+b\left|u_{x}\right|^{2}\right)^{2}+\varepsilon\left\langle u_{t}, u\right\rangle+\frac{\varepsilon \delta}{2}\left|u_{x}\right|^{2} \\
& \geq(1-\varepsilon)\left|u_{t}\right|^{2}+(\alpha-\varepsilon)\left|u_{x x}\right|^{2} \\
& \geq \frac{1}{2} \min \{1, \alpha\}\left\|\left(u(t), u_{t}(t)\right)\right\|_{E}^{2} .
\end{aligned}
$$

Combining (22) with (21), we see that for any given bounded subset $Z$ of $E$, there exists a constant $\varepsilon=\varepsilon(n+\beta, Z)>0$, which can be chosen small enough so that

$$
C(n+\beta) \varepsilon^{2}\left(C_{0}+C_{1}\left\|\left(u_{0}, u_{1}\right)\right\|_{E}^{4}\right)+2 C(n+\beta) \varepsilon q C_{2}\left(u_{0}, u_{1}\right) \leq 1 .
$$

It follows that

$$
\frac{1}{2} \min \{1, \alpha\}\left\|\left(u(t), u_{t}(t)\right)\right\|_{E}^{2} \leq L(0) \exp \left(-\frac{\varepsilon}{2} t\right)+\frac{a^{2}}{b}+1,
$$


for $t \in I_{\max }$. This inequality shows that any mild solution $w(t)=\left(u(t), u_{t}(t)\right)$ cannot blow up. In other words, all the solutions exist globally for $t \in$ $[0, \infty)$. In addition,

$$
\limsup _{t \rightarrow \infty}\|w(t)\|_{E}^{2} \leq 2(\min \{1, \alpha\})^{-1}\left(a^{2} b^{-1}+1\right) .
$$

Hence the closed bounded ball

$$
\begin{aligned}
B_{r}=\{y \in E: & \left.\|y\|_{E} \leq r\right\}, \\
& \text { with constant } r>\left[2 \max \left\{1, \alpha^{-1}\right\}\left(a^{2} b^{-1}+1\right)\right]^{1 / 2},
\end{aligned}
$$

is an absorbing set for the solution semigroup $S(t)$. Here, as usual, the solution semigroup is defined by $S(t) w_{0}=w\left(t ; w_{0}\right)$ for $t \geq 0$ and $w_{0} \in E$.

Based on the dissipativity of this semiflow, one can explore the existence of a global attractor. However, since the spillover problem is not closely related to the global attractor even if it exists, we shall skip over the discussion about the global attractor and directly work on the existence of inertial manifolds in the next section.

\section{The Existence of Inertial Manifolds}

For the dynamical system $\vartheta$ in the space $E$ defined by the solution semigroup $\{S(t): t \geq 0\}$, a set $\mathbf{M} \subset E$ is called an inertial manifold for $\vartheta$, if $\mathbf{M}$ is a Lipschitz continuous finite-dimensional manifold, positively invariant under $S(t)$, and attracts all the trajectories at a locally uniform exponential rate. That is, for any given bounded subset $Z$ of $E$, there exist constants $\mu(Z)>0$ and $C(Z)>0$, such that

$$
\operatorname{dist}_{E}\left(w\left(t ; w_{0}\right), \mathbf{M}\right) \leq C(Z) \exp (-\mu(Z) t), \quad \text { for } t \geq 0, \quad \text { and any } w_{0} \in Z \text {. }
$$

Note that this definition is slightly generalized. Because usually it is required that an inertial manifold have a uniform exponential attracting rate $\mu>0$, but the coefficient $C(Z)$ can depend on the given bounded set $Z$ from which the trajectory is started (cf. [5]).

In this section, we shall prove that there exist inertial manifolds for the concerned dynamical system $\vartheta$ associated with this solution semigroup $\{S(t): t \geq 0\}$.

Let $H_{m}=\operatorname{Span}\left\{e_{1}, \ldots, e_{m}\right\}$ and $P_{m}: H \rightarrow H_{m}$ be the orthogonal projection from $H$ onto $H_{m}$. Then let

$$
\begin{gathered}
Q_{m}=I_{H}-P_{m} \\
\Pi_{m}=\operatorname{diag}\left(P_{m}, P_{m}\right): E \rightarrow H_{m} \times H_{m} \\
\Theta_{m}=I_{E}-\Pi_{m} .
\end{gathered}
$$


The decompositions $H=P_{m} H \oplus Q_{m} H$ and $E=\left(\Pi_{m} E\right) \oplus\left(\Theta_{m} E\right)$ are orthogonal direct sums of subspaces, in which $P_{m} H$ has a finite dimension $m$ and $\Pi_{m} E$ has a finite dimension $2 m$.

Accordingly, the $H$-valued function $u(t)$ admits an orthogonal decomposition $u(t)=p(t)+h(t)$, with $p(t)=P_{m} u(t)$ and $h(t)=Q_{m} u(t)$. The $E$-valued function $w(t)=\operatorname{col}(u(t), v(t))$ has an orthogonal decomposition $w(t)=\pi(t)+\vartheta(t)$, with $\pi(t)=\Pi_{m} w(t)$ and $\vartheta(t)=\Theta_{m}(t) w(t)$. Because of the commutativity between $A^{1 / 2}$ and $P_{m}$, the second-order evolution equation (4) can be decomposed into the following coupled equations:

$$
\begin{aligned}
\frac{d^{2} p}{d t^{2}}+\alpha A p & +\delta A^{1 / 2} \frac{d p}{d t} \\
& +\left[a+b\left|A^{1 / 4} u\right|^{2}+q\left\langle A^{1 / 2} u, u_{t}\right\rangle^{2(n+\beta)+1}\right] A^{1 / 2} p=0
\end{aligned}
$$

$$
\begin{aligned}
\frac{d^{2} h}{d t^{2}}+\alpha A h & +\delta A^{1 / 2} \frac{d h}{d t} \\
& +\left[a+b\left|A^{1 / 4} u\right|^{2}+q\left\langle A^{1 / 2} u, u_{t}\right\rangle^{2(n+\beta)+1}\right] A^{1 / 2} h=0,
\end{aligned}
$$

with initial data $p(0)=P_{m} u_{0}, p_{t}(0)=P_{m} u_{1}$, and $h(0)=Q_{m} u_{0}, h_{t}(0)=$ $Q_{m} u_{1}$, respectively. Define a functional $J_{u}(t)$ by

$$
J_{u}(t)=a+b\left|A^{1 / 4} u\right|^{2}+q\left\langle A^{1 / 2} u, u_{t}\right\rangle^{2(n+\beta)+1} .
$$

Theorem 3. There exists a flat inertial manifold $\mathbf{M}$ in E, given by

$$
\mathbf{M}=H_{m} \times H_{m},
$$

for the dynamical system $\vartheta$ generated by the solution semigroup of Equation (8), where $m>0$ is a suitably large number.

Proof. Obviously, the set $\mathbf{M}$ given by (29) is a finite-dimensional subspace, hence it is a Lipschitz continuous, linear manifold. This $\mathbf{M}$ is positively invariant under the semigroup $\{S(t): t \geq 0\}$, because of the commutativity between $A^{1 / 2}$ and $P_{m}$. In fact, if $w_{0}=\left(u_{0}, u_{1}\right) \in \mathbf{M} \subset D(G)$, the mild solution $w(t)=\left(u(t), u_{t}(t)\right.$ of Equation (8) is a classical solution, so that the first component $u(t)=p(t)+h(t)$ satisfies Equation (4).

Therefore, the functions $p(t)$ and $h(t)$ are respectively classical solutions of Equation $(27)_{p}$ and Equation $(27)_{h}$, with $h(0)=h_{t}(0)=0$. By the uniqueness of solutions of Equation $(27)_{h}$, it follows that $h(t)=0$, for $t \geq 0$, and consequently $p(t)$ is the solution of

$$
\begin{aligned}
\frac{d^{2} p}{d t^{2}}+\alpha A p & +\delta A^{1 / 2} \frac{d p}{d t} \\
& +\left[a+b\left|A^{1 / 4} p\right|^{2}+q\left\langle A^{1 / 2} p, p_{t}\right\rangle^{2(n+\beta)+1}\right] A^{1 / 2} p=0,
\end{aligned}
$$




$$
p(0)=u_{0} \in H_{m}, \quad p_{t}(0)=u_{1} \in H_{m} .
$$

This proves the positive invariance of $\mathbf{M}$.

It remains to prove that $\mathbf{M}$ is exponentially attracting. By taking the inner product in $H$ of Equation $(27)_{h}$ with $2 h_{t}+\xi h$, where $\xi$ is an undetermined constant, we obtain

$$
\begin{aligned}
& \frac{d}{d t}\left\{\left|h_{t}\right|^{2}+\alpha\left|h_{x x}\right|^{2}+\xi\left\langle h_{t}, h\right\rangle+(\xi \delta / 2)\left|h_{x}\right|^{2}\right\} \\
& \quad+\left\{2 \delta\left|h_{x t}\right|^{2}-\xi\left|h_{t}\right|^{2}+\xi \alpha\left|h_{x x}\right|^{2}\right\} \\
& \quad+\left\{2 J_{u}(t)\left\langle h_{x}, h_{x t}\right\rangle+\xi J_{u}(t)\left|h_{x}\right|^{2}\right\} \\
& =0 .
\end{aligned}
$$

By Theorem 2 and its proof, we know that, for every given bounded set $Z$ in $E$ and for any initial point $w_{0} \in Z$, the trajectory $w\left(t ; w_{0}\right)$ will enter the fixed absorbing ball $B_{r}$ in $E$ (and stay in it forever) at an exponential rate $\varepsilon(n+\beta, Z) / 2$ after a transient period $\left[0, t_{0}\right]$ with $t_{0}=t_{0}(Z)$ also depending on $Z$. For this reason, below we assume that the trajectories have already been staying in the fixed absorbing ball $B_{r}$ after the transient period. Then we have

$$
\begin{aligned}
\left|J_{u}(t)\right| & \leq\left.|a+b| u_{x}\right|^{2}-q\left\langle u_{x x}, u_{t}\right\rangle^{2(n+\beta)+1} \mid \\
& \leq|a|+b\left\|w\left(t ; w_{0}\right)\right\|_{E}^{2}+q\left\|w\left(t ; w_{0}\right)\right\|_{E}^{4(n+\beta)+2} \\
& \leq|a|+b r^{2}+q r^{4(n+\beta)+2}, \quad \text { for } t \geq t_{0},
\end{aligned}
$$

where $r$ is the radius of the ball $B_{r}$ in (26). By using (32), we can estimate the last two terms in (31) as follows. Let

$$
\Pi(r)=|a|+b r^{2}+q r^{4(n+\beta)+2} .
$$

Then,

$$
\begin{aligned}
\mid 2 J_{u}(t)\left\langle h_{x}, h_{x t}\right\rangle & +\xi J_{u}(t)\left|h_{x}\right|^{2} \mid \\
& \leq 2 \Pi(r)\left|h_{x}\right|\left|h_{x t}\right|+\xi \Pi(r)\left|h_{x}\right|^{2} \\
& \leq\left[[\Pi(r)]^{2} \delta^{-1}+\xi \Pi(r)\right]\left|h_{x}\right|^{2}+\delta\left|h_{x t}\right|^{2} \\
& \leq \frac{[\Pi(r)]^{2} \delta^{-1}+\xi \Pi(r)}{\sqrt{\lambda_{m+1}}}\left|h_{x x}\right|^{2}+\delta\left|h_{x t}\right|^{2} \\
& \leq K(r, \xi)(m+1)^{-2} \pi^{-2}\left|h_{x x}\right|^{2}+\delta\left|h_{x t}\right|^{2},
\end{aligned}
$$

where $K(r, \xi)=[\Pi(r)]^{2} \delta^{-1}+\xi \Pi(r)$. Substituting (34) into (31), we obtain

$$
\begin{aligned}
& \frac{d}{d t}\left\{\left|h_{t}\right|^{2}+\alpha\left|h_{x x}\right|^{2}+\xi\left\langle h_{t}, h\right\rangle+(\xi \delta / 2)\left|h_{x}\right|^{2}\right\} \\
& \quad+\left\{\delta\left|h_{x t}\right|^{2}-\xi\left|h_{t}\right|^{2}+\xi \alpha\left|h_{x x}\right|^{2}-K(r, \xi)(m+1)^{-2} \pi^{-2}\left|h_{x x}\right|^{2}\right\} \\
& \quad \leq 0 .
\end{aligned}
$$


Let

$$
Y(t)=\left|h_{t}\right|^{2}+\alpha\left|h_{x x}\right|^{2}+\xi\left\langle h_{t}, h\right\rangle+(\xi \delta / 2)\left|h_{x}\right|^{2}
$$

and

$$
\Phi(t)=\delta\left|h_{x t}\right|^{2}-\xi\left|h_{t}\right|^{2}+\xi \alpha\left|h_{x x}\right|^{2}-K(r, \xi)(m+1)^{-2} \pi^{-2}\left|h_{x x}\right|^{2} .
$$

Then we have

$$
\begin{aligned}
\Phi(t)- & (\xi / 2) Y(t) \geq \delta\left|h_{x t}\right|^{2}-(3 \xi / 2)\left|h_{t}\right|^{2}+(\xi \alpha / 2)\left|h_{x x}\right|^{2} \\
& -K(r, \xi)(m+1)^{-2} \pi^{-2}\left|h_{x x}\right|^{2}-(\xi / 2)\left\langle h_{t}, h\right\rangle \\
\geq & (\delta-2 \xi)\left|h_{x t}\right|^{2} \\
& +\left\{(\xi \alpha / 2)-[(\xi / 2)+K(r, \xi)](m+1)^{-2} \pi^{-2}\right\}\left|h_{x x}\right|^{2} \\
\geq & 0 .
\end{aligned}
$$

Now choose $\xi$ and fix it, such that

$$
0<\xi \leq \min \left\{1, \alpha(1+\delta)^{-1}, \delta / 2\right\},
$$

and then take a positive integer $m$ large enough so that

$$
m \geq-1+\sqrt{\left(\xi \alpha \pi^{2}\right)^{-1}[\xi+2 K(r, \xi)]} .
$$

By these choices, it follows that

$$
\frac{d}{d t} Y(t)+\frac{\xi}{2} Y(t) \leq 0, \quad \text { for } t \geq t_{0},
$$

so that

$$
\begin{aligned}
& \frac{1}{2} \min \{1, \alpha\}\left\|\left(\begin{array}{c}
h(t) \\
h_{t}(t)
\end{array}\right)\right\|_{E}^{2} \\
& =\frac{1}{2} \min \{1, \alpha\}\left(\left|h_{t}\right|^{2}+\left|h_{x x}\right|^{2}\right) \\
& \leq \frac{1}{2} \min \{1, \alpha\}\left(\left|h_{t}\right|^{2}+\left|h_{x x}\right|^{2}\right)+\frac{1-\xi}{2}\left|h_{t}\right|^{2}+\frac{1}{2}(\alpha-\xi(1+\delta))\left|h_{x x}\right|^{2} \\
& \leq\left|h_{t}\right|^{2}+\alpha\left|h_{x x}\right|^{2}+\xi\left\langle h_{t}, h\right\rangle+(\xi \delta / 2)\left|h_{x}\right|^{2} \\
& =Y(t) \\
& \leq Y\left(t_{0}\right) \exp \left(-\frac{\xi}{2}\left(t-t_{0}\right)\right) \\
& \leq[1+\alpha+\xi+(\xi \delta / 2)]\left\|\left(\begin{array}{c}
h\left(t_{0}\right) \\
h_{t}\left(t_{0}\right)
\end{array}\right)\right\|_{E}^{2} \exp \left(-\frac{\xi}{2}\left(t-t_{0}\right)\right) \\
& \leq(2+\alpha+\delta)\left\|\left(\begin{array}{c}
u\left(t_{0}\right) \\
u_{t}\left(t_{0}\right)
\end{array}\right)\right\|_{E}^{2} \exp \left(-\frac{\xi}{2}\left(t-t_{0}\right)\right) \\
& \leq(2+\alpha+\delta) r^{2} \exp \left(-\frac{\xi}{2}\left(t-t_{0}\right)\right), \quad \text { for } t \geq t_{0} .
\end{aligned}
$$


Hence,

$$
\begin{aligned}
& \|\vartheta(t)\|_{E}^{2}=\left\|\Theta_{m} w(t)\right\|_{E}^{2}=\left\|\left(\begin{array}{c}
h(t) \\
h_{t}(t)
\end{array}\right)\right\|_{E}^{2} \\
& \leq 2 \min \{1, \alpha\}^{-1}(2+\alpha+\delta)\left\|\left(\begin{array}{c}
u\left(t_{0}\right) \\
u_{t}\left(t_{0}\right)
\end{array}\right)\right\|_{E}^{2} \exp \left(-\frac{\xi}{2}\left(t-t_{0}\right)\right) \\
& \leq 2 \min \{1, \alpha\}^{-1}(2+\alpha+\delta) r^{2} \exp \left(-\frac{\xi}{2}\left(t-t_{0}\right)\right), \quad \text { for } t \geq t_{0} .
\end{aligned}
$$

The inequality (43) is valid for all solutions with initial data $w_{0}$ in $E$.

Note that, by condition (39), the exponentially decaying rate $\xi$ depends only on the parameters $\alpha$ and $\delta$. So $\xi$ is independent of the specific bounded set $Z$ in which the initial point $w_{0}$ lies.

Also observe that, by condition (40), the dimension $m$ of the flat manifold $H_{m}$ depends only on the parameters $\{\alpha, \delta, a, b, q, n, \beta\}$ and the radius $r$ of the absorbing ball, where $r$ in turn is determined by $\{\alpha, a, b\}$. Therefore, the dimension $m$ is determined by the physical parameters of Equation (2).

Finally, (43) implies that, for $t \geq t_{0}$,

$$
\begin{aligned}
& \operatorname{dist}_{E}\left(w\left(t ; w_{0}\right), \mathbf{M}\right)=\operatorname{dist}_{E}(\pi(t)+\vartheta(t), \mathbf{M}) \leq\|\vartheta(t)\|_{E} \\
& \leq 2 \min \{1, \alpha\}^{-1}(2+\alpha+\delta)\left\|w\left(t_{0}\right)\right\|_{E}^{2} \exp \left(-\frac{\xi}{2}\left(t-t_{0}\right)\right) .
\end{aligned}
$$

From (24) we have

$$
\left\|w\left(t_{0}\right)\right\|_{E}^{2} \leq 2 \min \{1, \alpha\}^{-1}\left\{K_{1}(Z) \exp \left(-\frac{\varepsilon(Z)}{2} t_{0}\right)+\frac{a^{2}}{b}\right\},
$$

where $\varepsilon=\varepsilon(Z)$ depends on the specific bounded subset $Z$ of $E$ from which the trajectory is started, and $K_{1}(Z)=\sup \left\{L(0): w(0)=w_{0} \in Z\right\}$. Substituting (45) into (44) we have, for $t \geq t_{0}$,

$$
\begin{aligned}
\operatorname{dist}_{E}\left(w\left(t ; w_{0}\right)\right. & , \mathbf{M}) \leq 4 \min \{1, \alpha\}^{-1}(2+\alpha+\delta) \\
\cdot & \left\{K_{1}(Z) \exp \left(-\frac{\varepsilon(Z)}{2} t_{0}\right)+\frac{a^{2}}{b}\right\} \exp \left(-\frac{\xi}{2}\left(t-t_{0}\right)\right) .
\end{aligned}
$$

In order to take into account the behavior of the solutions in the transient period, let

$$
\mu=\mu(Z)=\frac{1}{2} \min \{\varepsilon(Z), \xi\}
$$

and

$$
K_{2}\left(Z, t_{0}(Z)\right)=4 \min \{1, \alpha\}^{-1}(2+\alpha+\delta)\left\{K_{1}(Z)+\frac{a^{2}}{b} \exp \left(\frac{\xi}{2} t_{0}\right)\right\} .
$$


Then we can conclude that

$$
\operatorname{dist}_{E}\left(w\left(t ; w_{0}\right), \mathbf{M}\right) \leq K_{2}\left(Z, t_{0}(Z)\right) \exp (-\mu(Z) t), \quad \text { for } t \geq 0 .
$$

Thus, by definition, $\mathbf{M}=H_{m} \times H_{m}$ is an inertial manifold with nonuniform exponential attracting rate for this dynamical system. The proof is completed.

We also obtain an explicit estimate for the dimension of such an inertial manifold. In fact,

$$
\operatorname{dim} \mathbf{M}=2 m,
$$

where $m$ is an integer satisfying the condition (40) in which $\xi$ satisfies (39). The following is a concrete estimate for this dimension in terms of the physical parameters involved in this nonlinear beam equation.

Corollary 4. Let $m$ be the smallest positive integer which satisfies

$$
\begin{aligned}
m & \geq-1 \\
& +\frac{1}{\pi \alpha^{1 / 2}} \sqrt{1+2 \rho(\alpha, a, b, q, n, \beta)\left[1+\delta^{-1} \rho(\alpha, a, b, q, n, \beta) \sigma(\alpha, \delta)\right]},
\end{aligned}
$$

where

$$
\begin{aligned}
\rho(\alpha, a, b, q, n, \beta)= & |a|+2 a^{2} \max \left\{1, \alpha^{-1}\right\} \\
& +1+q\left[2 a^{2} b^{-1} \max \left\{1, \alpha^{-1}\right\}+1\right]^{2(n+\beta)+1}
\end{aligned}
$$

and

$$
\sigma(\alpha, \delta)=\max \left\{1, \alpha^{-1}(1+\delta), 2 \delta^{-1}\right\} .
$$

Then there exists an inertial manifold $\mathbf{M}$ given by (29) with $\operatorname{dim} \mathbf{M}=2 \mathrm{~m}$.

Proof. Letting $r^{2}=2 a^{2} b^{-1} \max \left\{1, \alpha^{-1}\right\}+1$ and $\xi=\min \left\{1, \alpha(1+\delta)^{-1}\right.$, $\delta / 2\}$ in $\Pi(r)$ and $K(r, \xi)$, we see that formula (40) can be written as (51). So the result holds.

\section{Remarks.}

Here we have the following two remarks. First, from (51), we see that as the coefficient $\delta$ of the structural damping becomes smaller, the lower bound for the dimension of an inertial manifold will increase and its growth rate is roughly proportional to $\delta^{-1}$, provided that there are no dramatic changes in the other parameters.

Second and more important, if one does not know each parameter exactly and must allow the value variations over some moderate range, then (51) also gives a conservative estimate of the lower bound for the dimension of an inertial manifold. Together with the result presented in the next section, this will be useful in providing a robust stabilization of this nonlinear beam system by a finite-dimensional, linear feedback control.

The governing equation for the dynamics on an inertial manifold is called inertial form, which is simply a system of ordinary differential equations. 
Corollary 5. For the inertial manifold $\mathbf{M}=H_{m} \times H_{m}$ the inertial form is the following equation in the subspace $H_{m}$.

$$
\begin{aligned}
& \frac{d^{2} p}{d t^{2}}+\alpha A p(t) \\
& +\delta A^{1 / 2} \frac{d p}{d t}+\left[a+b\left|A^{1 / 4} p(t)\right|^{2}+q\left\langle A^{1 / 2} p(t), \frac{d p}{d t}\right\rangle\right] A^{1 / 2} p(t) \\
& =0, \quad t \geq 0, \\
& \quad p(0)=p_{0} \in H_{m}, \quad p_{t}(0)=p_{1} \in H_{m} .
\end{aligned}
$$

Here $p(t)$ is a finite-dimensional vector.

\section{Stabilization by Finite-Dimensional Feedback Control}

In the last section we shall resolve the spillover problem based on the existence of inertial manifolds. This approach for achieving stabilization is potentially applicable to other distributed control systems featuring nonlocal nonlinearities.

Now consider the full equation, Equation (1), with the control function $f(t, x)$ on the right-hand side. The stabilization problem for Equation (1) is to find a linear or nonlinear feedback mapping $F: E \rightarrow H$, in general it can be nonautonomous, such that the feedback control

$$
f(t, \cdot)=F\left(u(t), u_{t}(t)\right), \quad t \geq 0,
$$

makes the closed-loop system asymptotically stable in the sense that all the mild solutions $w(t)$ of the closed-loop equation converge to zero in $E$, as $t \rightarrow \infty$. If so, Equation (1) is said to be strongly stabilizable by the feedback control. Moreover, if in addition the convergence occurs at a uniform exponential rate, then Equation (1) is called uniformly exponentially stabilizable. If the convergence occurs at a nonuniform exponential rate, which means that the decay rate depends on the bounded set which the initial data belong to, then Equation (1) is said to be nonuniformly exponentially stabilizable.

Theorem 6. The control system Equation (1) is nonuniformly exponentially stabilizable by a finite-dimensional linear feedback control

$$
f(t, \cdot)=a A^{1 / 2} P_{m} u(t), \quad t \geq 0,
$$

where $P_{m}: H \rightarrow H_{m}$ is the orthogonal projection, and $H_{m}$ is the factor subspace associated with the inertial manifold $\mathbf{M}=H_{m} \times H_{m}$ for the uncontrolled Equation (2).

Proof. By applying this feedback control (55) to Equation (1) and decomposing the equation into two component equations according to the decomposition of $H=H_{m} \oplus Q_{m} H$, we can get

$$
p_{t t}+\alpha p_{x x x x}-\delta p_{x x t}-\left(J_{u}(t)-a\right) p_{x x}=0,
$$


$(56)_{h}$

$$
h_{t t}+\alpha h_{x x x x}-\delta h_{x x t}-J_{u}(t) h_{x x}=0
$$

where $J(u(t))$ is defined by $(28)$, and $u(t)=p(t)+h(t)$ is a solution of the following closed-loop equation

$$
\begin{aligned}
& u_{t t}+\alpha A u+ \delta A^{1 / 2} u_{t} \\
&+ {\left[a+b\left|A^{1 / 4} u\right|^{2}+q\left\langle A^{1 / 2} u, u_{t}\right\rangle^{2(n+\beta)+1}\right] A^{1 / 2} u } \\
&= a A^{1 / 2} P_{m} u(t), \\
& u(0)=u_{0}, \quad u_{t}(0)=u_{1} .
\end{aligned}
$$

Since this linear feedback (55) only partially cancels the term $-a p_{x x}$ on the left-hand side of the equation (56) or (57), an easy adaptation in the corresponding proof assures us that Theorem 2 remains valid and the ball $B_{r}$ remains an absorbing set for the new closed-loop equation, Equation (57).

Since the linear feedback (55) does not affect Equation $(56)_{h}$, the same argument used in the proof of Theorem 3 for the exponential attraction, especially within the absorbing ball $B_{r}$, of the manifold $\mathbf{M}$ remains true without any change in the constants. Hence, it is true that

$$
\left\|\left(\begin{array}{c}
h(t) \\
h_{t}(t)
\end{array}\right)\right\|_{E}^{2} \leq K_{2}\left(Z, t_{0}(Z)\right) \exp (-\mu(Z) t), \quad t \geq 0,
$$

where $h(t)=Q_{m} u(t)$ and the constants $K_{2}\left(Z, t_{0}(Z)\right)$ and $\mu(Z)$ are the same as before.

It suffices to concentrate on Equation $(56)_{p}$. Specifically, we want to prove that the component $p(t)=P_{m} u(t)$ of the solution $u(t)$ of the closed-loop equation (57) also converges to zero at nonuniform exponential rate. By taking the inner product of $(56)_{p}$ in $H$ with $2 p_{t}+\kappa p$, where $\kappa$ is an undetermined constant, we get

$$
\begin{aligned}
& \frac{d}{d t}\left\{\left|p_{t}\right|^{2}+\alpha\left|p_{x x}\right|^{2}+\kappa\left\langle p_{t}, p\right\rangle+(\kappa \delta / 2)\left|p_{x}\right|^{2}+(b / 2)\left|p_{x}\right|^{4}\right\} \\
& \quad+\left\{2 \delta\left|p_{x t}\right|^{2}-\kappa\left|p_{t}\right|^{2}+\kappa \alpha\left|p_{x x}\right|^{2}+\kappa b\left|p_{x}\right|^{4}\right\} \\
& \quad+\left\{2 b\left\langle p_{x x}, p_{t}\right\rangle\left|h_{x}\right|^{2}+\kappa b\left|p_{x}\right|^{2}\left|h_{x}\right|^{2}\right. \\
& \left.\quad+q\left[2\left\langle p_{x t}, p_{x}\right\rangle+\kappa\left|p_{x}\right|^{2}\right]\left\langle u_{x}, u_{x t}\right\rangle^{2(n+\beta)+1}\right\}
\end{aligned}
$$




$$
\begin{aligned}
= & \frac{d}{d t}\left\{\left|p_{t}\right|^{2}+\alpha\left|p_{x x}\right|^{2}+\kappa\left\langle p_{t}, p\right\rangle+(\kappa \delta / 2)\left|p_{x}\right|^{2}+(b / 2)\left|p_{x}\right|^{4}\right\} \\
& +\left\{2 \delta\left|p_{x t}\right|^{2}-\kappa\left|p_{t}\right|^{2}+\kappa \alpha\left|p_{x x}\right|^{2}+\kappa b\left|p_{x}\right|^{4}\right. \\
& \left.+\left[2 q\left\langle p_{x}, p_{x t}\right\rangle^{2}+\kappa q\left|p_{x}\right|^{2}\left\langle p_{x}, p_{x t}\right\rangle\right]\left\langle u_{x}, u_{x t}\right\rangle^{2(n+\beta)}\right\} \\
& +\left\{2 b\left\langle p_{x x}, p_{t}\right\rangle\left|h_{x}\right|^{2}+\kappa b\left|p_{x}\right|^{2}\left|h_{x}\right|^{2}\right. \\
& \left.+q\left[2\left\langle p_{x t}, p_{x}\right\rangle+\kappa\left|p_{x}\right|^{2}\right]\left\langle h_{x}, h_{x t}\right\rangle\left\langle u_{x}, u_{x t}\right\rangle^{2(n+\beta)}\right\} \\
= & 0 .
\end{aligned}
$$

Let

$$
\Gamma(t)=\left|p_{t}\right|^{2}+\alpha\left|p_{x x}\right|^{2}+\kappa\left\langle p_{t}, p\right\rangle+(\kappa \delta / 2)\left|p_{x}\right|^{2}+(b / 2)\left|p_{x}\right|^{4},
$$

and

$$
\begin{aligned}
\Delta(t)= & 2 \delta\left|p_{x t}\right|^{2}-\kappa\left|p_{t}\right|^{2}+\kappa \alpha\left|p_{x x}\right|^{2}+\kappa b\left|p_{x}\right|^{4} \\
& +\left[2 q\left\langle p_{x}, p_{x t}\right\rangle^{2}+\kappa q\left|p_{x}\right|^{2}\left\langle p_{x}, p_{x t}\right\rangle\right]\left\langle u_{x}, u_{x t}\right\rangle^{2(n+\beta)} .
\end{aligned}
$$

Using Young's inequality to treat the last term of $\Delta(t)$, we have

$$
\begin{aligned}
\Delta(t) & -\frac{\kappa}{2} \Gamma(t) \geq \delta\left|p_{x t}\right|^{2}+\left(\delta-\frac{3 \kappa}{2}-\frac{\kappa^{2}}{2}\right)\left|p_{t}\right|^{2} \\
+ & \kappa\left(\frac{\alpha}{2}-\frac{\kappa}{2}-\frac{\kappa \delta}{4}\right)\left|p_{x x}\right|^{2} \\
& +\left[\frac{3 \kappa b}{4}\left|p_{x}\right|^{4}+2 q\left\langle p_{x}, p_{x t}\right\rangle^{2}\left\langle u_{x}, u_{x t}\right\rangle^{2(n+\beta)}\right] \\
& -\eta \kappa q\left|p_{x}\right|^{4}\left\langle u_{x}, u_{x t}\right\rangle^{2(n+\beta)}-C(\eta) \kappa q\left|p_{x}\right|^{2}\left|p_{x t}\right|^{2}\left\langle u_{x}, u_{x t}\right\rangle^{2(n+\beta)} \\
\geq & \left(\delta-\frac{3 \kappa}{2}-\frac{\kappa^{2}}{2}\right)\left|p_{t}\right|^{2}+\kappa\left(\frac{\alpha}{2}-\frac{\kappa}{2}-\frac{\kappa \delta}{4}-\eta q r^{4(n+\beta)+2}\right)\left|p_{x x}\right|^{2} \\
& +\left[\delta-C(\eta) \kappa q r^{4(n+\beta)+2}\right]\left|p_{x t}\right|^{2} \\
\geq & 0, \quad \text { for } t \geq 0,
\end{aligned}
$$

if we choose $\eta>0$ and $\kappa>0$ small enough, so that

$$
\frac{\alpha}{4}-\eta q r^{4(n+\beta)+2} \geq 0,
$$

and

$$
\begin{aligned}
\delta-\frac{3 \kappa}{2}-\frac{\kappa^{2}}{2} \geq 0, & \frac{\alpha}{2}-\kappa-\frac{\kappa \delta}{2} \geq 0, \quad \delta-\kappa C(\eta) q r^{4(n+\beta)+2} \geq 0 \\
& \min \{1, \alpha)-\kappa>0 .
\end{aligned}
$$


On the other hand, for the last $\{\ldots\}$ portion on the left-hand side of the last equality in (59), we have the following estimate, which is valid within the given absorbing ball $B_{r}$.

$$
\begin{aligned}
& \left.\left|2 b\left\langle p_{x x}, p_{t}\right\rangle\right| h_{x}\right|^{2}+\kappa b\left|p_{x}\right|^{2}\left|h_{x}\right|^{2} \\
& +q\left[2\left\langle p_{x t}, p_{x}\right\rangle+\kappa\left|p_{x}\right|^{2}\right]\left\langle h_{x}, h_{x t}\right\rangle\left\langle u_{x}, u_{x t}\right\rangle^{2(n+\beta)} \mid \\
& \leq(2+\kappa) b r^{2}\left|h_{x}\right|^{2}+(2+\kappa) q r^{4(n+\beta)+2}\left|h_{x x}\right|\left|h_{t}\right| \\
& \leq(2+\kappa) r^{2}\left(b+q r^{4(n+\beta)}\right) K_{2}\left(Z, t_{0}(Z)\right) \exp (-\mu(Z) t), \quad \text { for } t \geq t_{0} \text {, }
\end{aligned}
$$

where we used the fact that $\left|p_{x x}\right|,\left|p_{x}\right|,\left|p_{t}\right|,\left|u_{x x}\right|,\left|u_{x}\right|,\left|u_{t}\right| \leq r$ in the fixed absorbing ball $B_{r}$ and, in the last inequality of (65), $\left|h_{x}\right|^{2}$ and $\left|h_{x x}\right|\left|h_{t}\right|$ are replaced by the estimate(58) for the $h$-component.

We now substitute (62) and (65) into (59), then get

$$
\frac{d}{d t} \Gamma(t)+\frac{\kappa}{2} \Gamma(t) \leq K_{3}\left(Z, t_{0}(Z)\right) \exp (-\mu(Z) t), \quad t \geq t_{0}
$$

where

$$
K_{3}\left(Z, t_{0}(Z)\right)=(2+\kappa) r^{2}\left(b+q r^{4(n+\beta)}\right) K_{2}\left(Z, t_{0}(Z)\right),
$$

with $\kappa$ chosen and fixed as above. By integrating this inhomogeneous differential inequality (66) over the time interval $\left[t_{0}, t\right]$, we obtain

$$
\begin{aligned}
\Gamma(t) \leq & \Gamma\left(t_{0}\right) \exp \left(-\frac{\kappa}{2}\left(t-t_{0}\right)\right) \\
& +\frac{K_{3}\left(Z, t_{0}(Z)\right)}{\left|\frac{\kappa}{2}-\mu(Z)\right|} \exp \left(-\min \left\{\frac{\kappa}{2}, \mu(Z)\right\}\left(t-t_{0}\right)\right), \quad t \geq t_{0},
\end{aligned}
$$

if $\frac{\kappa}{2} \neq \mu(Z)$, or

$(68)_{b}$

$$
\begin{aligned}
\Gamma(t) \leq & \Gamma\left(t_{0}\right) \exp \left(-\frac{\kappa}{2}\left(t-t_{0}\right)\right) \\
& +K_{3}\left(Z, t_{0}(Z)\right)\left(t-t_{0}\right) \exp \left(-\frac{\kappa}{2}\left(t-t_{0}\right)\right) \\
\leq & \frac{4}{\kappa} K_{3}\left(Z, t_{0}(Z)\right) \exp \left(-\frac{\kappa}{4}\left(t-t_{0}\right)\right), \quad t \geq t_{0},
\end{aligned}
$$

if $\frac{\kappa}{2}=\mu(Z)$. By $(47), \mu(Z)=(1 / 2) \min \{\varepsilon(Z), \xi\}$. Now let

$$
\nu=\nu(Z)=\frac{1}{2} \min \left\{\frac{\kappa}{2}, \varepsilon(Z), \xi\right\}
$$


Since

$$
\begin{gathered}
\Gamma(t) \geq \frac{1}{2} \min \{1, \alpha\}\left(\left|p_{t}\right|^{2}+\left|p_{x x}\right|^{2}\right)=\frac{1}{2} \min \{1, \alpha\}\left\|\left(\begin{array}{c}
p(t) \\
p_{t}(t)
\end{array}\right)\right\|_{E}^{2}, \\
\Gamma\left(t_{0}\right) \leq\left(2+\kappa+\frac{\delta}{2}\right) r^{2}+\frac{b}{2} r^{4}
\end{gathered}
$$

where we can take $r^{2}=2 a^{2} b^{-1} \max \left\{1, \alpha^{-1}\right\}+1$ in (26). Let

$$
\begin{aligned}
K_{4}\left(Z, t_{0}(Z)\right)= & 2 \max \left\{1, \alpha^{-1}\right\}\left[\left(2+\alpha+\frac{\delta}{2}\right) r^{2}+\frac{b}{2} r^{4}\right. \\
& \left.+\frac{K_{3}\left(Z, t_{0}(Z)\right)}{\left|\frac{\kappa}{2}-\mu(Z)\right|}\right] \exp \left(\nu(Z) t_{0}\right), \quad \text { if } \frac{\kappa}{2} \neq \mu(Z),
\end{aligned}
$$

or

$$
\begin{aligned}
K_{4}\left(Z, t_{0}(Z)\right)= & 2 \max \left\{1, \alpha^{-1}\right\}\left[\left(2+\alpha+\frac{\delta}{2}\right) r^{2}+\frac{b}{2} r^{4}\right. \\
& \left.+\frac{4}{\kappa} K_{3}\left(Z, t_{0}(Z)\right)\right] \exp \left(\nu(Z) t_{0}\right), \quad \text { if } \frac{\kappa}{2}=\mu(Z) .
\end{aligned}
$$

Then from $(68)_{a}$ and $(68)_{b}$ it follows that

$$
\left\|\left(\begin{array}{c}
p(t) \\
p_{t}(t)
\end{array}\right)\right\|_{E}^{2} \leq K_{4}\left(Z, t_{0}(Z)\right) \exp (-\nu(Z) t), \quad t \geq t_{0}
$$

Next we combine this result with the exponential decay estimate (24) in the transient period $\left[0, t_{0}\right]$, which gives

$$
\begin{aligned}
& \left\|\left(\begin{array}{c}
p(t) \\
p_{t}(t)
\end{array}\right)\right\|_{E}^{2} \leq\left\|\left(\begin{array}{c}
u(t) \\
u_{t}(t)
\end{array}\right)\right\|_{E}^{2} \\
& \leq 2 \max \left\{1, \alpha^{-1}\right\}\left[L(0) \exp \left(-\frac{\varepsilon}{2} t\right)+\frac{a^{2}}{b}+1\right] \\
& \leq 2 \max \left\{1, \alpha^{-1}\right\}\left[K_{1}(Z)+\left(\frac{a^{2}}{b}+1\right) \exp \left(\frac{\varepsilon}{2} t_{0}\right)\right] \exp \left(-\frac{\varepsilon}{2} t\right) \\
& \leq K_{5}\left(Z, t_{0}(Z)\right) \exp (-\nu(Z) t), \text { for } t \in\left[0, t_{0}\right],
\end{aligned}
$$

where

$$
K_{5}\left(Z, t_{0}(Z)\right)=2 \max \left\{1, \alpha^{-1}\right\}\left[K_{1}(Z)+\left(\frac{a^{2}}{b}+1\right) \exp \left(\frac{\varepsilon}{2} t_{0}\right)\right] .
$$


Therefore, we have proved the nonuniform exponential decay of the $p$ component of the closed-loop solution, which is given by

$$
\left\|\left(\begin{array}{c}
p(t) \\
p_{t}(t)
\end{array}\right)\right\|_{E}^{2} \leq\left[K_{4}\left(Z, t_{0}(Z)\right)+K_{5}\left(Z, t_{0}(Z)\right)\right] \exp (-\nu(Z) t), \quad t \geq 0 .
$$

Finally, (58) and (76) together imply that the solution of the closed-loop equation (57) has the following property of nonuniform exponential decay,

$$
\left\|\left(\begin{array}{c}
u(t) \\
u_{t}(t)
\end{array}\right)\right\|_{E}^{2} \leq K_{6}(Z) \exp (-\nu(Z) t), \quad t \geq 0
$$

where

$$
K_{6}(Z)=\max \left\{K_{2}\left(Z, t_{0}(Z)\right), K_{4}\left(Z, t_{0}(Z)\right)+K_{5}\left(Z, t_{0}(Z)\right)\right\}
$$

Summary Remarks. As a conclusion, below we briefly summarize the important points in this paper.

First, Theorem 6 shows that, by using the finite-dimensional linear feedback (55), the exponential decay rate $\nu(Z)$ and the coefficient $K_{6}(Z)$ can be estimated in terms of the physical parameters in this beam equation and the radius of the initial bounded set $Z$ in $E$.

Second, the number $m$ of the modes involved in this stabilizing feedback can be estimated by Corollary 4 . Note that $m$ increases (roughly proportionally to $1 / \delta$ ) as the coefficient $\delta$ of the structural damping decreases.

Third, it is noteworthy that if we replace the physical parameters $\{\alpha, \delta, a$, $b, q, n, \beta\}$ in the dimension formula (51)-(52) by their conservative bounds of uncertainty, then Theorem 3, Corollary 4, and Theorem 6 regarding the existence of inertial manifolds, the dimension estimate, and the exponential stabilization are all robust. Therefore, in other words, we have obtained a robust solution to the concerned spillover problem.

Fourth, the results on the existence of inertial manifolds and on the finitedimensional stabilization hold also for the same nonlinear beam equation except that the structural damping $-\delta u_{x x t}$ is replaced by a strong structural damping $\delta u_{x x x x t}$ without any substantial change in the argument.

\section{REFERENCES}

1. A. V. Balakrishnan and L. W. Taylor, Distributed parameter nonlinear damping models for flight structures, in Proceedings "Damping '89", Flight Dynamics Lab and Air Force Wright Aeronautical Labs, WPAFB, 1989.

2. R. W. Bass and D. Zes, Spillover, nonlinearity, and flexible structures, in The Fourth NASA Workshop on Computational Control of Flexible Aerospace Systems, NASA Conference Publication \#10065 (ed. L. W. Taylor), 1991, 1-14.

3. A. M. Bloch and E. S. Titi, On the dynamics of rotating elastic beams, in Proceedings of the Conference on New Trends in Systems Theory (ed. G. Conti et al), Birkhäuser, 1990. 
4. I. D. Chueshov, Inertial manifolds in the problem of nonlinear vibrations of an infinite panel, Ukrainian Math. J. 42 (1990), 1291-1293.

5. C. Foias, G. R. Sell and R. Temam, Inertial manifolds for evolutionary equations, J. Differential Equations 73 (1988), 309-353.

6. J. M. Ghidaglia and A. Marzocchi, Long time behavior of strongly damped wave equations, global attractors and their dimensions, SIAM J. Math. Anal. 22 (1991), 879-895.

7. J. Hale, Asymptotic Behaviour of Dissipative Systems, Mathematical Surveys and Monographs, 25, Amer. Math. Soc., Providence, Rhode Island, 1988.

8. D. Sevcovic, Existence and limiting behavior for damped nonlinear evolution equations with nonlocal terms, Comment. Math. Univ. Carolinae 31 (1990), 283-293.

9. R. Temam, Infinitie Dimensional Dynamical Systems in Mechanics and Physics, Springer-Verlag, New York, 1988.

10. E. S. Titi, On approximate inertial manifolds to the Navier-Stokes equations, J. Math. Anal. Appl. 149 (1990), 540-557.

11. Y. You, Inertial manifolds and stabilization of nonlinear elastic systems with structural damping, in Differential Equations and Mathematical Physics (ed. W. Ames, E. Harrell and J. Herod), Academic Press, New York, 1993, 335-346.

12. Y. You, Spillover problem and global dynamics of nonlinear beam equations, in Differential Equations, Dynamical Systems, and Control Science, (ed. K. Elworthy, W. Everitt and E. B. Lee), Marcel Dekker, New York, 1993, 891-912.

Department of Mathematics, University of South Florida, Tampa, FL 33620-5700, USA

E-mail address: you@gauss.math.usf.edu 


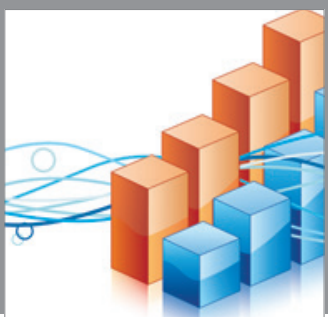

Advances in

Operations Research

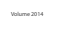

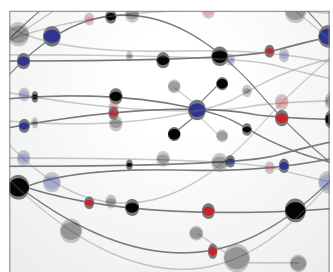

\section{The Scientific} World Journal
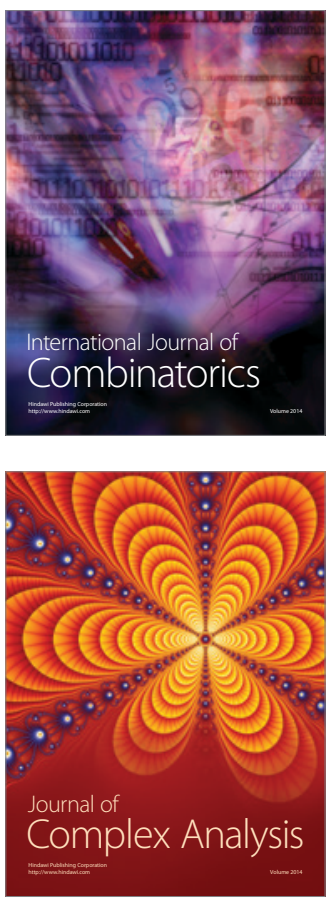

International Journal of

Mathematics and

Mathematical

Sciences
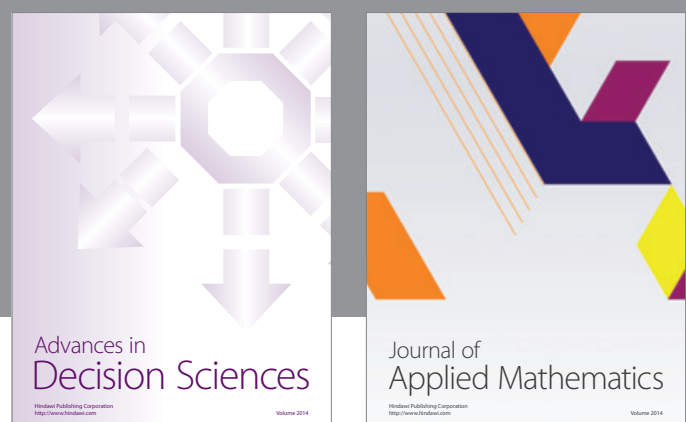

Journal of

Applied Mathematics
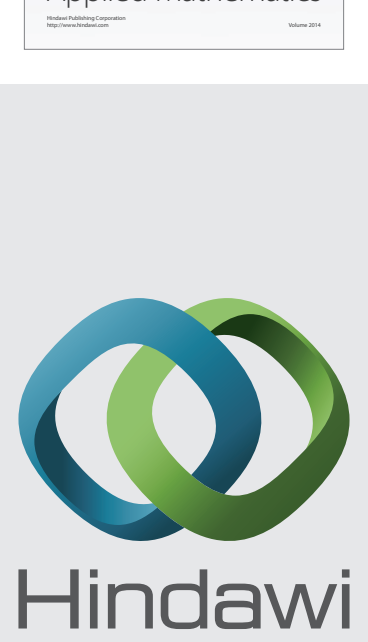

Submit your manuscripts at http://www.hindawi.com
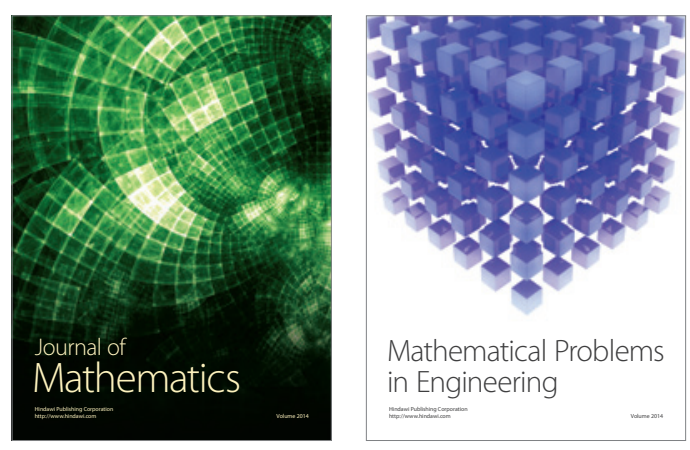

Mathematical Problems in Engineering
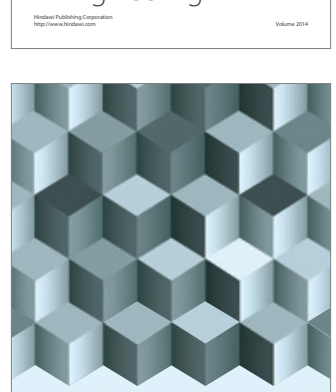

Journal of

Function Spaces
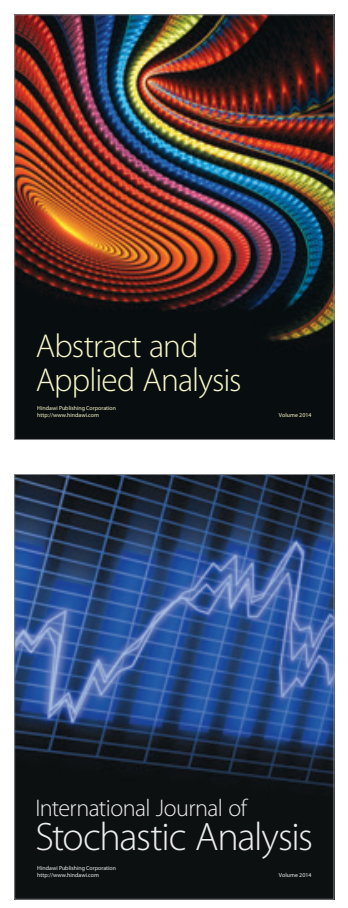

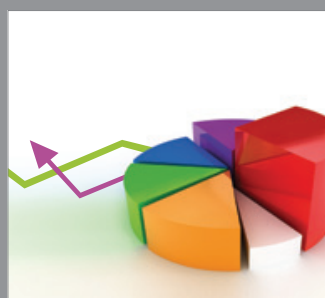

ournal of

Probability and Statistics

Promensencen
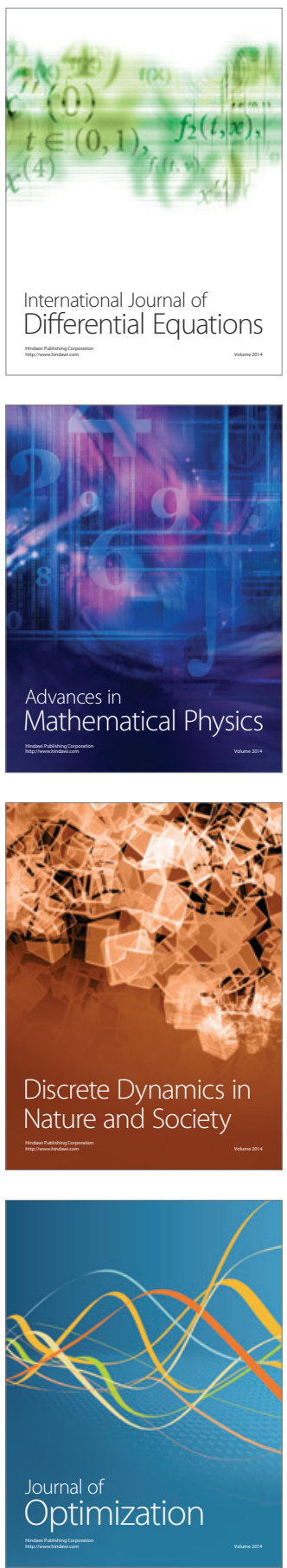